\title{
The UV-upturn in brightest cluster galaxies
}

\author{
S. I. Loubser ${ }^{1}$ and P. Sánchez-Blázquez ${ }^{2}$ \\ ${ }^{1}$ Centre for Space Research, North-West University, Potchefstroom 2520, South Africa \\ email: Ilani.Loubser@nwu.ac.za \\ ${ }^{2}$ Departamento de Física Teórica, Universidad Autónoma de Madrid, E28049, Spain \\ email: psanchezblazquez@googlemail.com
}

\begin{abstract}
This study is part of a series devoted to the investigation of a large sample of brightest cluster galaxies (BCGs), their properties and the relationships between these and the properties of the host clusters. In this paper, we compare the stellar population properties derived from high signal-to-noise, optical long-slit spectra with the GALEX ultraviolet (UV) colour measurements for 36 nearby BCGs to understand the diversity in the most rapidly evolving feature in old stellar systems, the UV-upturn. We investigate: (1) the possible differences between the UV-upturn of BCGs and those of a control sample of ordinary ellipticals in the same mass range, as well as possible correlations between the UV-upturn and other general properties of the galaxies; (2) possible correlations between the UV-upturn and the properties of the host clusters; (3) recently proposed scenarios where helium-sedimentation in the cluster centre can produce an enhanced UV-upturn. We find systematic differences between the UV-colours of BCGs and ordinary ellipticals, but we do not find correlations between these colours and the properties of the host clusters. Furthermore, the observations do not support the predictions made by the helium-sedimentation model as an enhancer of the UV-upturn.
\end{abstract}

Keywords. galaxies: evolution - galaxies: elliptical and lenticular, $\mathrm{cD}$ - galaxies: stellar content - ultraviolet: galaxies

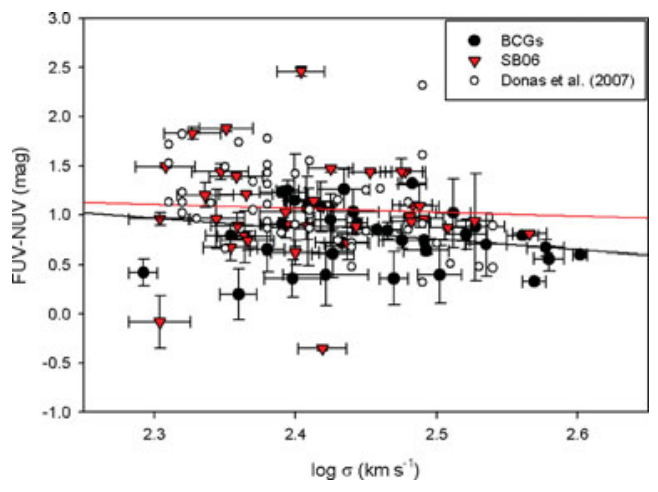

Figure 1. The top panel shows the FUV-NUV colour against velocity dispersion for BCGs and non-BCGs (see Loubser \& Sánchez-Blázquez 2011). The BCGs are indicated with the filled circles, and the non-BCGs are indicated with the filled triangles and empty circles. The correlation for the BCGs is the lower line of the two lines, the upper line is the correlation for non-BCGs. We find that the UV colour distributions of the two elliptical samples agree very well, whereas the distribution of the BCG sample is significantly different from both elliptical samples.

\section{Reference}

Loubser, S. I. \& Sánchez-Blázquez, P 2011, MNRAS, 410, 2679 